\title{
Solitary lung metastasis from primary prostate cancer with normal prostate specific antigen levels: A case report and literature review
}

\author{
NATSUMI MARU ${ }^{1}$, ASAKO OKABE ${ }^{2}$, HARUAKI HINO ${ }^{1}$, TAKAHIRO UTSUMI ${ }^{1}$, HIROSHI MATSUI ${ }^{1}$, \\ YOHEI TANIGUCHI ${ }^{1}$, TOMOHITO SAITO ${ }^{1}$, KOJI TSUTA ${ }^{2}$ and TOMOHIRO MURAKAWA ${ }^{1}$ \\ Departments of ${ }^{1}$ Thoracic Surgery and ${ }^{2}$ Pathology and Laboratory Medicine, \\ Kansai Medical University Hospital, Hirakata, Osaka 573-1191, Japan
}

Received October 27, 2021; Accepted December 7, 2021

DOI: 10.3892/wasj.2021.139

\begin{abstract}
Pulmonary involvement from prostate cancer is a well-known condition; however, solitary lung metastasis is rare, with its associated clinical characteristics not yet fully elucidated. The present study describes the case of a 77-year-old male, who had undergone radical prostatectomy at a previous hospital for primary prostatic carcinoma 14 years prior, who presented with a low-grade fever. At the previous hospital, salvage radiation therapy was being considered, as the prostate-specific antigen (PSA) level had gradually increased within the normal range. Computed tomography performed at the authors' institution revealed a solitary nodule with a spiculated morphology located on the upper lobe of the left lung, while positron emission tomography suggested malignancy without metastasis. Based on these findings, primary lung cancer was suspected and thoracoscopic left upper lobectomy with lymph node dissection was performed. The pathological diagnosis of the tumor was a solitary lung metastasis of prostate cancer. The post-operative recovery was uneventful. In addition to reporting a case with normal PSA levels, the present study also performed a literature review. According to previous case reports, there are some pitfalls of prostate cancer leading to misdiagnosis as a primary lung tumor. However, it is considered that surgical resection is associated with an increased diagnostic accuracy and long-term survival.
\end{abstract}

\section{Introduction}

Pulmonary metastasis secondary to prostate cancer has been reported in $>40 \%$ of patients in an autopsy series (1). By

Correspondence to: Dr Natsumi Maru, Department of Thoracic Surgery, Kansai Medical University Hospital, 2-3-1 Shin-machi, Hirakata, Osaka 573-1191, Japan

E-mail: marunat@hirakata.kmu.ac.jp

Key words: solitary lung metastasis, lung metastasectomy, prostate cancer contrast, solitary lung metastasis from prostate cancer is rare, and only 34 cases have been reported to date (Table I) (2-25). More than half of the patients (22/34) underwent lung resection, and the majority achieved cancer control and long-term survival.

The present study reports the 35th rare case of isolated lung metastasis from prostate cancer with normal prostate-specific antigen (PSA) levels 14 years after radical prostatectomy. In addition, a review of former case series was performed.

\section{Case report}

A 77-year-old male who was a previous smoker with low-grade fever was referred to Kansai Medical University Hospital. The patient had a medical history of primary prostate cancer, pathological stage T2bN0 [Gleason score (GS): 3+4] organ-confined disease. He underwent radical prostatectomy at a former hospital when he was 63 years of age. Following surgical treatment, the patient was followed-up without additional treatment. The patient's PSA level fell to undetectable levels for 11 years post-surgery. During his consultation, the patient's PSA level gradually increased and exceeded $0.2 \mathrm{ng} / \mathrm{ml}$ on two occasions without evidence of macroscopic recurrence. This condition is identified as biochemical recurrence. The gradual increase in the PSA levels continued on for a further 2 years. When the levels reached $0.412 \mathrm{ng} / \mathrm{ml}$, the doubling time was determined to be 2 years, and the imaging examination of metastasis prior to salvage radiotherapy was considered at the former hospital. Upon referral to Kansai Medical University Hospital due to low-grade fever, computed tomography (CT) revealed a small nodule $(1.5 \mathrm{~cm}$ in diameter) with a spiculated morphology and pleural indentation in the upper lobe of the left lung (Fig. 1A). Subsequently, 18-fluoro-2-deoxyglucose positron emission tomography $\left({ }^{18} \mathrm{~F}-\mathrm{FDG}-\mathrm{PET}\right)$ revealed highly selective accumulation in the nodule, strongly suggestive of a malignant tumor (maximum standardized uptake value, 3.6) (Fig. 1B). The CT scan and ${ }^{18}$ F-FDG-PET did not reveal any abnormal accumulation in the other organs. Primary lung cancer (clinical T1bN0M0) was highly suspected. The levels of serum tumor biomarkers for primary lung cancer were within the normal ranges: Carcinoembryonic antigen, $<1.0 \mathrm{ng} / \mathrm{ml}$ (normal range, 
$<5.0 \mathrm{ng} / \mathrm{ml})$; neuron-specific enolase, $8.2 \mathrm{ng} / \mathrm{ml}(<16.3 \mathrm{ng} / \mathrm{ml})$; and pro-gastrin-releasing peptide, $50.9 \mathrm{pg} / \mathrm{ml}(<81.0 \mathrm{pg} / \mathrm{ml})$. A thoracoscopic left upper lobectomy with hilar and mediastinal lymph node dissection was performed as a radical surgery for primary lung cancer. The duration of the surgery was $161 \mathrm{~min}$ and blood loss was $106 \mathrm{ml}$. An intraoperative frozen section was not submitted for examination due to the high index of suspicion for primary lung cancer based on the preoperative imaging and serum PSA levels within the normal range, and the limitations brought about by the coronavirus disease 2019 pandemic.

A post-operative histological examination revealed adenocarcinoma. The tumor exhibited crowded small glands with an amphophilic cytoplasm and enlarged nuclei with visible nucleoli (Fig. 2A and C). Immunohistochemical staining was then performed using sections at a thickness of $3-4 \mu \mathrm{m}$. The primary antibodies used were the following: Anti-thyroid transcription factor-1 monoclonal mouse antibody (clone 8G7G3/1; cat. no. IR056; Dako; Agilent Technologies, Inc.), hepatocyte nuclear factor $4 \alpha$ monoclonal mouse antibody (clone H1415; cat. no. PP-H1415-00, Perseus Proteomics, Inc.), anti-human $\alpha$-methylacyl-CoA racemase monoclonal rabbit antibody (clone 13H4; cat. no. M361629-2, Dako; Agilent Technologies, Inc.). The secondary reaction was performed using a Ventana OptiView DAB universal kit (111427; Roche Diagnostics K.K.). Antibody incubations were performed at $72^{\circ} \mathrm{C}$ for $16 \mathrm{~min}$. No counterstain was used. The sections were examined under an Olympus BX51 microscope (Olympus Corporation). Immunohistochemical staining revealed thyroid transcription factor $1(-)$, hepatocyte nuclear factor $4 \alpha(-)$, PSA (+) and $\alpha$-methylacyl-CoA racemase (+) in the tumor cells (Fig. 2B and D). The tumor was diagnosed as differentiated adenocarcinoma metastatic from prostate cancer, based on hematoxylin and eosin staining and immunohistochemistry. Following surgery, the patient's recovery remained uneventful, and he was discharged on post-operative day 9. His serum PSA levels decreased to $0.07 \mathrm{ng} / \mathrm{ml}$. He was closely followed-up at the Department of Urology in Kansai Medical University Hospital for 1 year and 4 months without any additional post-operative treatment. No recurrent signs, including an elevation in PSA levels, have been detected thus far.

\section{Discussion}

Prostate cancer has a high risk of biochemical recurrence, with the incidence ranging from 27 to $53 \%$ (26). It has been demonstrated that the mean interval between biochemical recurrence (PSA re-elevation $>0.2 \mathrm{ng} / \mathrm{ml}$ twice following radical prostatectomy) and clinical recurrence (macroscopic appearance of the recurrent lesion that can be identified by imaging or histological examination) is $\sim 8$ years, suggesting that the long-term observation is required for patients with prostate cancer (27). For the patient presented herein, the possibility of a clinical recurrence should have been suspected when the CT scan revealed a lung nodule, as biochemical recurrence following radical prostatectomy was already observed in the previous 3 years. However, it was not suspected due to the following reasons: The CT findings of this case included a solitary lesion with a spiculated morphology, which is more typical of primary lung cancer, rather than a metastatic lung tumor, and the serum PSA level was still within the normal range. Additionally, solitary lung metastasis from prostate cancer is extremely rare. Previously, radiological evidence of pulmonary metastases following surgery, chemotherapy, or radiotherapy for prostate cancer was detected in 48 out of 1,290 patients $(3.6 \%)$ while a solitary pulmonary nodule was detected in only 11 out of 1,290 patients $(0.85 \%)$ (3). For these reasons, the clinical recurrence of prostate cancer was not strongly suspected preoperatively in the present case.

A search of PubMed and Ichuushi-web (http://www.jamas. or.jp) identified 24 articles and 34 patients with solitary lung metastasis from prostate cancer. A total of 22 articles described their cases in detail. The clinical characteristics of 23 cases were reviewed, including 22 from the literature and the present case (Table I). A review of a previous case series (4-25) helped to identify pitfalls which are prone to misinterpretation errors with significant consequences for patients.

First, the concept of the biochemical recurrence of prostate cancer is not a commonly known issue in the fields of surgery other than urology. However, considering the possibility of clinical recurrence from serum PSA fluctuations, even in patients with normal values as in the case presented herein, is crucial. It has been demonstrated that metastases from prostate cancer with normal serum PSA levels suggest high-grade cancer ( $\mathrm{GS} \geq 8$ ), small cell carcinoma, neuroendocrine tumor, or neuroendocrine differentiation (28). Among the prior 23 cases, high-grade cancer or neuroendocrine differentiation was found in 9 patients, and in the majority of cases (8/9), the serum PSA levels were within the normal range. However, even among patients with low-grade cancer, normal serum PSA levels were found in more than half of the cases (6/10). Of all the cases, 74\% (17/23) had isolated lung metastasis with normal PSA levels, regardless of the histology.

The standard procedure for pulmonary metastases is partial resection aimed at the preservation of lung parenchyma. Among the reviewed 23 cases with solitary lung metastasis, 22 patients had undergone lung resection and 14 of them reported satisfactory outcomes. Lobectomy was performed in 9 patients, including the present case. The reasons for this procedure in some cases are highly suggestive. In four cases, intraoperative frozen section examinations were unable to determine whether the tumor was a metastasis or primary lung adenocarcinoma $(6,15,18,25)$. Considering these previous case studies, there is a high likelihood of the misdiagnosis of prostatic adenocarcinoma with lung metastasis as a primary pulmonary neoplasm in clinical practice, and this is considered to be a second pitfall. Copeland et al (29) reported the presence of a tubule-papillary or a carcinoid-like histologic pattern in a pulmonary tumor which was not associated with the histological features of prostate carcinoma (29). Thus, in clinical practice, it is prudent to consider solitary lung metastasis from prostate cancer as a differential diagnosis for patients previously treated for prostate cancer.

However, the surgical resection of isolated lung metastasis from prostate cancer can be justified by its satisfactory outcomes. Pulmonary metastatic lesions which underwent metastasectomies have been closely related to survival in various types of cancer, including colon and uterine cervical cancer $(30,31)$. The number of metastases from prostate cancer 


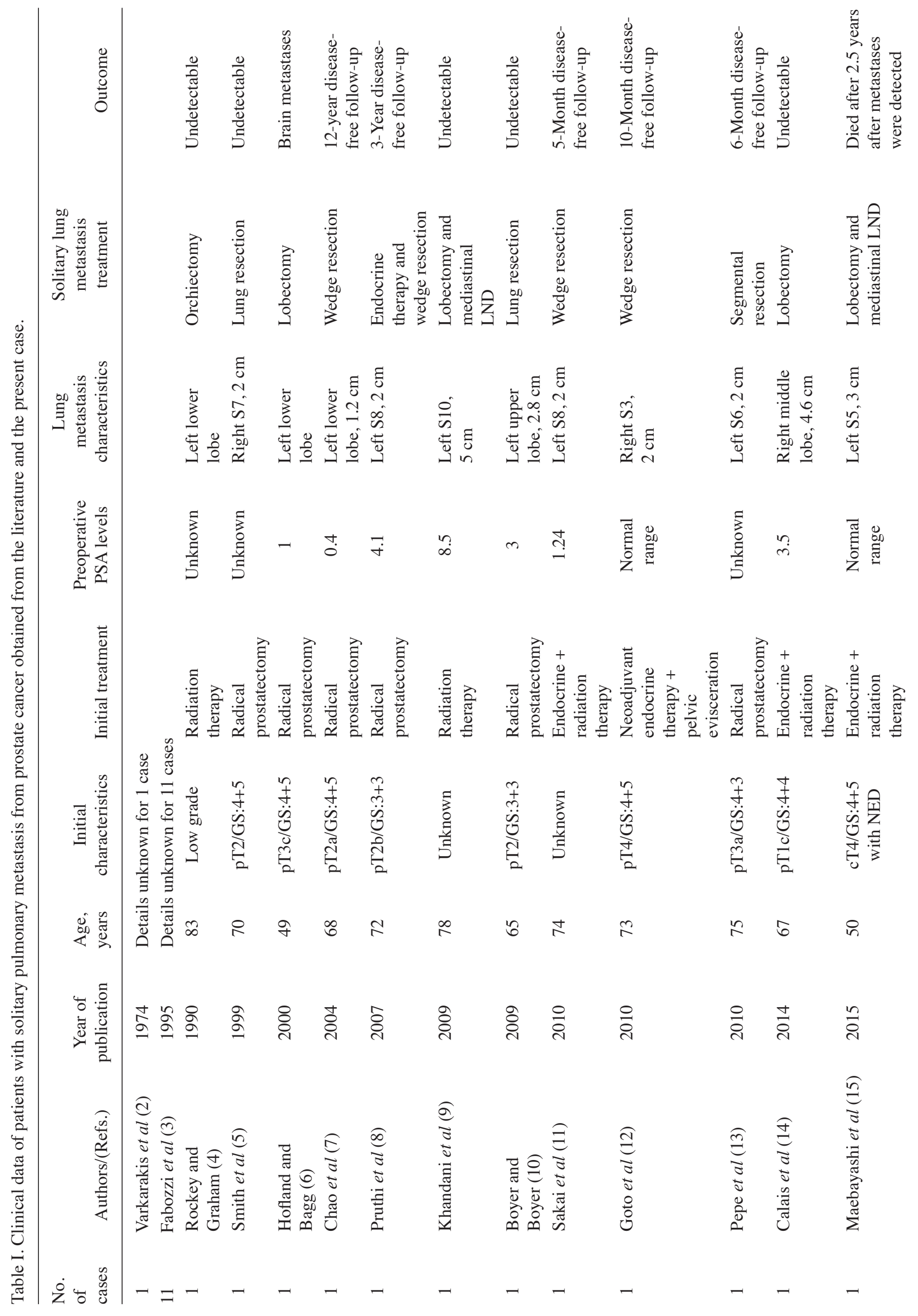




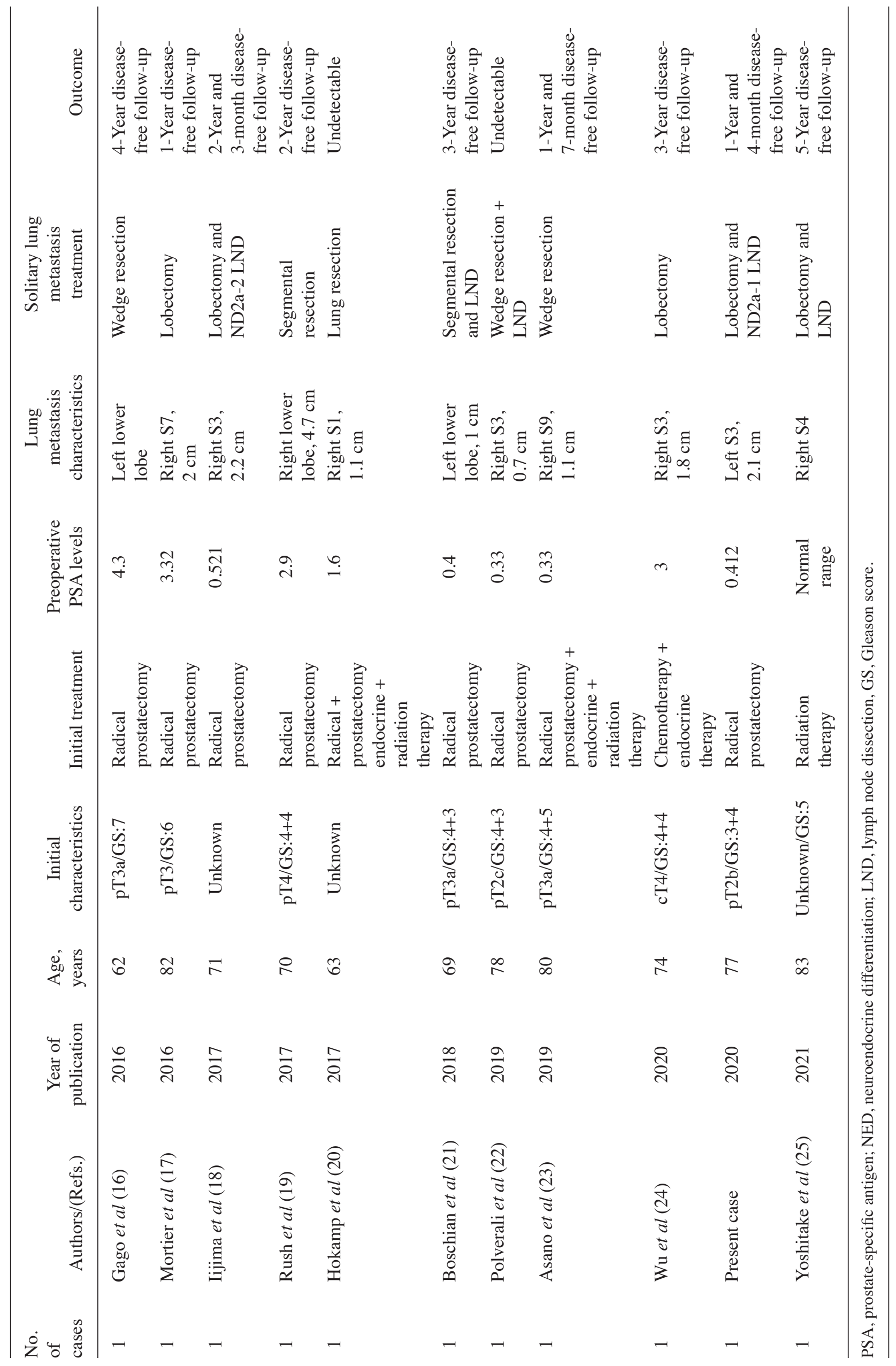



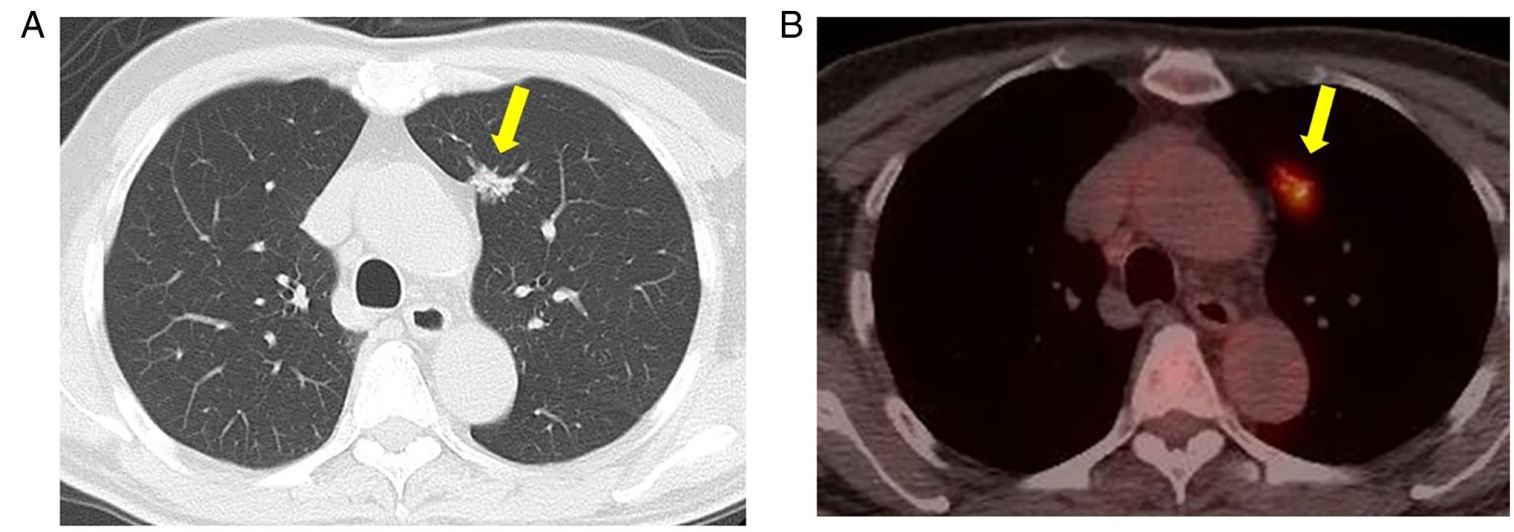

Figure 1. Preoperative computed tomography and 18-fluoro-2-deoxyglucose positron emission tomography. (A) Computed tomography revealed a small nodule with a spiculated morphology and pleural indentation $1.5 \mathrm{~cm}$ in dimeter located in the left upper lobe (yellow arrow). (B) 18-fluoro-2-deoxyglucose positron emission tomography revealed high accumulation in the nodule (yellow arrow), strongly suggestive of a malignant tumor (maximum standardized uptake value, 3.6).

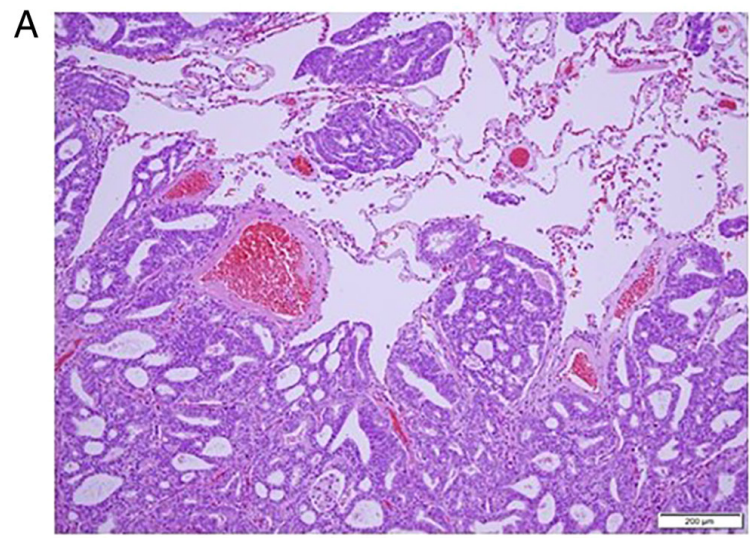

B
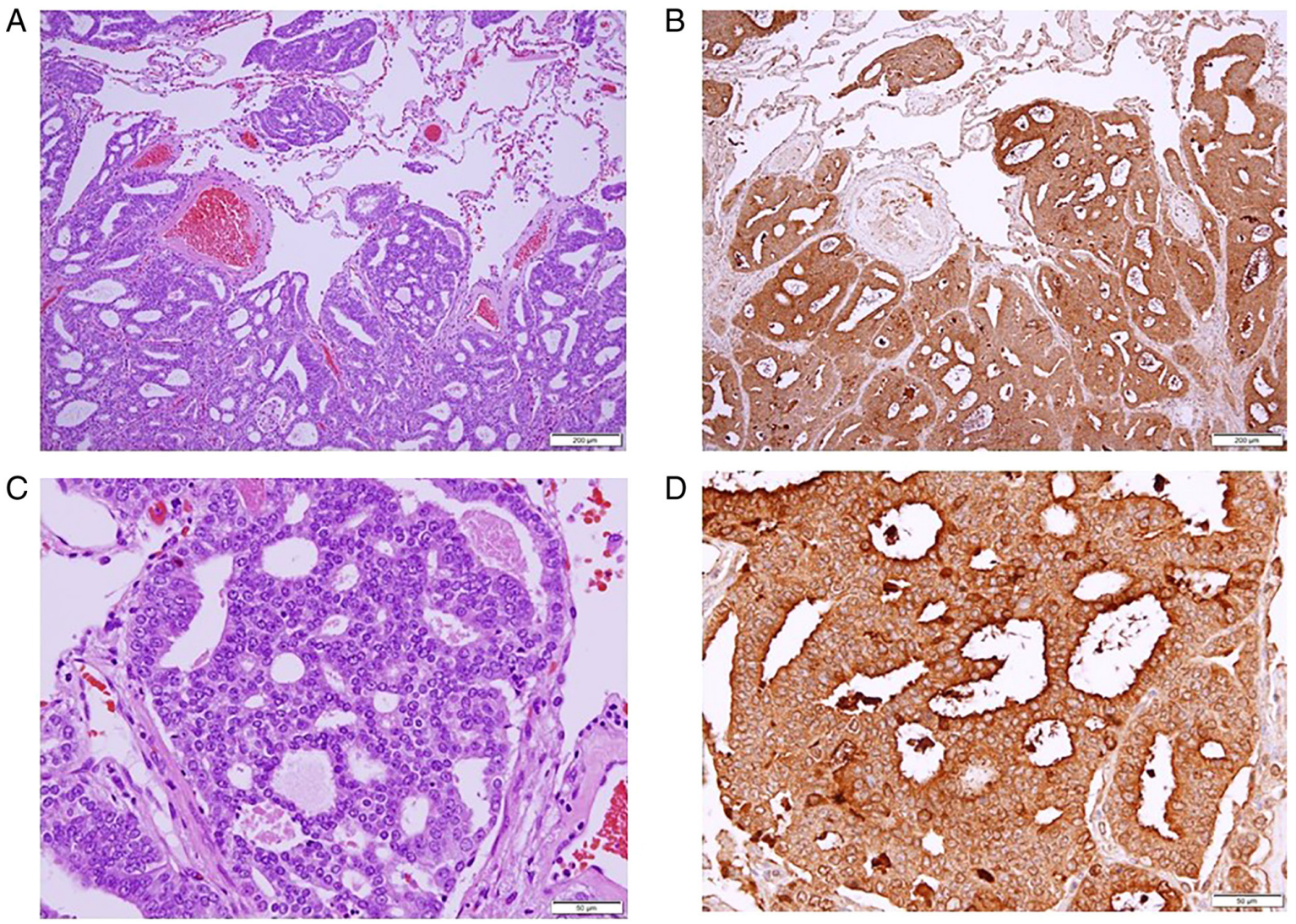

Figure 2. Histological examination of the lung tumor. Hematoxylin and eosin staining at a magnification of (A) x100 and (C) x400. Immunohistochemical staining of prostate-specific antigen revealed adenocarcinoma derived from prostate cancer. Magnification: (B) x100 (D) x400.

is considered an independent prognostic factor affecting the 5 -year cancer-specific survival rate, of which characteristic is the same as other malignancies previously mentioned. The 5-year cancer-specific survival rate for patients with one metastasis is $90 \%$, compared to $32 \%$ for those with two or more (32). Surgical resection, particularly for solitary pulmonary lesions, is associated with favorable outcomes in patients with prostate cancer.

In conclusion, as demonstrated in the present study, there are some pitfalls of solitary lung metastasis from prostate cancer leading to misdiagnosis as a primary lung cancer and occasionally they were treated with surgical resection including lobectomy. However, the favorable prognosis in previous case series suggests that surgical resection may be justified.

\section{Acknowledgements}

Not applicable.

\section{Funding}

No funding was received. 


\section{Availability of data and materials}

The datasets used and/or data analyzed during the present study are available from the corresponding author on reasonable request.

\section{Authors' contributions}

NM and TM wrote the manuscript. NM and TM performed the surgery (a thoracoscopic left upper lobectomy with hilar and mediastinal lymph node dissection). NM, TU, HM, YT, TS, HH and TM determined the treatment plan. AO and KT performed the pathological diagnosis and contributed to the drafting of the pathological findings section of the manuscript. $\mathrm{NM}$ and $\mathrm{HH}$ confirm the authenticity of all the raw data. All authors have read and approved the final manuscript.

\section{Ethics approval and consent to participate}

The present study was approved by the Ethics Committee of Kansai Medical University Hospital (approval no. 2015630). The patient provided written informed consent.

\section{Patient consent for publication}

The patient provided consent for the publication of his data and associated images.

\section{Competing interests}

The authors declare that they have no competing interests.

\section{References}

1. Bubendorf L, Schopfer A, Wagner U, Sauter G, Moch H, Willi N, Gasser TC and Mihatsch MJ: Metastatic patterns of prostate cancer: An autopsy study of 1589 patients. Hum Pathol 31: 578-583, 2000.

2. Varkarakis MJ, Winterberger AR, Gaeta J, Moore RH and Murphy GP: Lung metastases in prostatic carcinoma. Clinical significance. Urology 3: 447-452, 1974.

3. Fabozzi SJ, Schellhammer PF and El-Mahdi AM: Pulmonary metastases from prostate cancer. Cancer 75: 2706-2709, 1995.

4. Rockey KE and Graham TE: Prostate adenocarcinoma metastatic to the lung. Postgrad Med 87: 199-205, 1990.

5. Smith CP, Sharma A, Ayala G, Cagle P and Kadmon D: Solitary pulmonary metastasis from prostate cancer. J Urol 162: 2102, 1999.

6. Hofland CA and Bagg MD: An isolated pulmonary metastasis in prostate cancer. Mil Med 165: 973-974, 2000.

7. Chao DH, Higgins JP and Brooks JD: Biochemical remission after resection of prostate cancer lung metastasis. Urology 63 584-585, 2004.

8. Pruthi RS, Hubbard JS, Kouba E and Wallen E: Androgenindependent prostate cancer treated with resection of the solitary metastatic site. Urol Int 79: 371-373, 2007.

9. Khandani AH, Funkhouser WK, Feins R and Socinski MA: Simultaneous FDG PET +/Glut1+ lung and FDG PET-/Glut1subcarinal lymph node metastases from prostate cancer. Ann Nucl Med 23: 595-597, 2009

10. Boyer BP and Boyer MJ: An elusive tumor in a man who has evidence of prostate cancer metastasis. JAAPA 22: 22-25, 2009.

11. Sakai T, Kimura D, Hatanaka R, Yamada Y, Tsushima T, Fukuda I and Kamata Y1: Surgical treated pulmonary metastasis from prostatic cancer: Report of a case. Kyobu Geka 63: 340-343, 2010 (In Japanese).

12. Goto T, Maeshima A, Oyamada Y and Kato R: Solitary pulmonary metastasis from prostate sarcomatoid cancer. World J Surg Oncol 8: 101, 2010.

13. Pepe P, Fraggetta F, Tornabene F, Nicolosi M and Aragona F: Solitary lung metastasis after radical prostatectomy in presence of undetectable PSA. Arch Ital Urol Androl 84: 208-210, 2010.
14. Calais J, Lussato D, Menard J, Kerviler ED, Mongiat-Artus P, Castier Y and Merlet P: Resection of a solitary pulmonary metastasis from prostatic Adenocarcinoma misdiagnosed as a Bronchocele: Usefulness of 18F-choline and 18F-FDG PET/CT J Thorac Oncol 12: 1826-1829, 2014.

15. Maebayashi T, Abe K, Aizawa T, Sakaguchi M, Ishibashi N, Fukushima S, Honma T, Kusumi Y, Matsui T and Kawata N: Solitary pulmonary metastasis from prostate cancer with neuroendocrine differentiation: A case report and review of relevant cases from the literature. World J Surg Oncol 13: 173-180, 2015.

16. Gago JP, Câmara G, Dionísio J and Opinião A: Pulmonary metastasis as sole manifestation of relapse in previously treated localized prostate cancer: Three exceptional case reports. Ecancermedicalscience 10: 645, 2016.

17. Mortier D, Baten E, Vandeurzen K and van Renterghem K: The benefit of a surgical resection of a solitary pulmonary metastasis of prostate cancer after radical prostatectomy. Curr Urol 20: 210-212, 2016.

18. Iijima $Y$, Kinoshita $H$, Nakajima $Y$, Akiyama $H$, Uramoto $H$ and Hirata T: Isolated solitary lung metastasis 12 years after radical prostatectomy for prostate cancer without elevation of the serum prostate-specific antigen level. Jpn J Chest Surg 31: 648-652, 2017 (In Japanese).

19. Rush J, Pai R and Parikh RA: Complete biochemical response after pulmonary metastasectomy in prostate adenocarcinoma. Exp Hematol Oncol 6: 25, 2017.

20. Hokamp NG, Kobe C, Linzenich E, Maintz D and Drzezga A: Solitary PSMA-positive pulmonary metastasis in biochemical relapse of prostate cancer. Clin Nucl Med 42: 406-407, 2017.

21. Boschian R, Rizzo M, Zandona L, Trombetta C and Liguori G: Pulmonary recurrence from prostate cancer and biochemical remission after metastasis directed therapy. A case report. Arch Ital Urol Androl 90: 74-75, 2018.

22. Polverari G, Ceci F, Allen-Auerbach M, Gupta P, Fishbein MC, Reiter RE, Lee JM, Hope TA, Carroll RM, Czernin J and Calais J: Solitary mucinous prostate adenocarcinoma lung metastasis detected by ${ }^{68} \mathrm{Ga}$-PSMA-11 PET/CT. Clin Genitourin Cancer 17: e53-e55, 2019.

23. Asano H, Arakawa S, Kato D, Mori S, Nakada T and Ohtsuka T: A case of single lung metastasis with normal serum PSA level after prostate cancer surgery. Jpn Chest Surg 33: 66-69, 2019 (In Japanese).

24. Wu LX, Lei L, Zhu YC, Du KQ, Li XF, Chen HF, Wang WX and $\mathrm{Xu}$ CW: A prostate cancer patient with isolated lung metastases: A case report. Transl Cancer Res 9: 2064-2068, 2020.

25. Yoshitake H, Oura S, Yamaguchi T and Makimoto S: Solitary lung metastasis of prostate cancer with a long disease-free interval and normal prostate-specific antigen level. Case Rep Oncol 14: 284-289, 2021.

26. Heidenreich A, Bastian PJ, Bellmunt J, Bolla M, Joniau S, van der Kwast T, Mason M, Matveev V, Wiegel T, Zattoni F, et al: EAU guidelines on prostate cancer. Part II: Treatment of advanced, relapsing, and castration-resistant prostate cancer. Eur Urol 65: 467-479, 2014.

27. Tourinho-Barbosa R, Srougi V, Nunes-Silva I, Baghdadi M, Rembeyo G, Eiffel SS, Barret E, Rozet F, Galiano M, Cathelineau X and Sanchez-Salas R: Biochemical recurrence after radical prostatectomy: What does it mean? Int Braz J Urol 44: 14-21, 2018.

28. Leibovici D, Spiess PE, Agarwal PK, Tu SM, Pettaway CA, Hitszhusen K, Millikan RE and Pisters L: Prostate cancer progression in the presence of undetectable or low serum prostate-specific antigen level. Cancer 109: 198-204, 2007.

29. Copeland JN, Amin BD, Humphrey PA, Tamboli P, Ro JY and Gal AA: The morphologic spectrum of metastatic prostatic adenocarcinoma to the lung. Am J Clin Pathol 117: 552-557, 2002.

30. Onaitis MW, Petersen RP, Haney JC, Salts L, Park B, Flores R, Rizk N, Bains MS, Dycoco J, D'Amico TA, et al: Prognostic factors for recurrence after pulmonary resection of colorectal cancer metastases. Ann Thorac Surg 87: 1684-1688, 2009.

31. Yamamoto K, Yoshikawa H, Shiromizu K, Saito T, Kuzuya K, Tsunematsu R and Kamura T: Pulmonary metastasectomy for uterine cervical cancer: A multivariate analysis. Ann Thorac Surg 77: 1179-1182, 2004.

32. Ost P, Decaestecker K, Lambert B, Fonteyne V, Delrue L, Lumen N, Ameye F and De Meerleer G: Prognostic factors influencing prostate cancer-specific survival in non-castrate patients with metastatic prostate cancer. Prostate 74: 297-305, 2014.

This work is licensed under a Creative Commons Attribution-NonCommercial-NoDerivatives 4.0 International (CC BY-NC-ND 4.0) License. 